\title{
Characteristics and outcomes of a multi-institution cohort of infants with necrotizing enterocolitis totalis
}

\author{
Allison T. Rose $\mathbb{D}^{1 \times}$, Isabella Zaniletti $\mathbb{D}^{2}$, Matthew T. Santore $\mathbb{I D}^{3}$ and Anthony J. Piazza $\mathbb{D}^{1}$ \\ (c) The Author(s), under exclusive licence to Springer Nature America, Inc. 2021
}

\begin{abstract}
OBJECTIVE: To characterize the presentation, management and outcomes of infants with necrotizing enterocolitis totalis ( $t$ NEC) vs surgical non-totalis NEC (sNEC).

STUDY DESIGN: This retrospective study identified infants undergoing surgery for NEC through The Children's Hospitals Neonatal Database. Demographic, surgical and mortality characteristics were compared.

RESULTS: Of 1059 infants, 161 (15.2\%) had $t$ NEC. Perinatal characteristics did not differ. $t$ NEC infants were older and were less likely to have pneumoperitoneum at referral $(5.6 \%$ vs $13.1 \%, p<0.001)$ or intestinal perforation at surgery $(38.5 \%$ vs $66.7 \%, p<0.001)$. Infants with $t$ NEC were more acidotic preoperatively $(7.1$, [IQR 7, 7.3] vs 7.3, [IQR 7.2, 7.4], $p<0.001)$. Mortality was $96.9 \%$ for $t$ NEC and $26.5 \%$ for $s$ NEC $(p<0.001)$. $t$ NEC cases varied by center, accounting for $0-43 \%$ of all surgical NEC cases.

CONCLUSIONS: Mortality is high for tNEC infants, who present at older age, with greater illness severity but are less likely to have intestinal perforation than sNEC infants.
\end{abstract}

Journal of Perinatology (2022) 42:440-445; https://doi.org/10.1038/s41372-021-01191-7

\section{INTRODUCTION}

Ten percent of neonatal intensive care deaths are attributed to necrotizing enterocolitis (NEC) [1]. Necrotizing enterocolitis totalis $(t N E C)$, a rare form of NEC characterized by near-total bowel necrosis, is associated with almost $100 \%$ fatality [2]. Of infants who require surgery for NEC, $11 \%$ have $t$ NEC [3], and, amongst those who die from NEC, 33\% have total bowel necrosis[1]. However, the true incidence of this disease is not known, as $t$ NEC cannot currently be preoperatively identified and is subjectively defined in the literature $[2,4]$.

Death from NEC has been associated with lower gestational age, lower birth weight, need for mechanical ventilation at diagnosis, and black race [5]. Although risk factors for NEC have been the subject of significant research [6], little is understood about which infants are susceptible to $t N E C$. Describing this unique cohort of infants is challenging, as single institutions may have few patients with the disease, and infants may die without surgery or require transfer for surgical care. Thus, the understanding of $t$ NEC is limited to studies with small sample size $[4,7,8]$. As neonatal care advances and intestinal rehabilitation care improves, there is growing interest in the management and outcomes of infants with $t$ NEC $[2,9-11]$. The Children's Hospitals Neonatal Database (CHND) provides prospectively collected data on rare conditions including $t$ NEC, allowing for better understanding of disease and the potential for outcomes prediction [12]. The primary purpose of this study was to describe the presentation at surgical referral of a large national cohort of $t$ NEC infants and to compare presentation, surgical management, and mortality of $t$ NEC infants to infants who require surgery for NEC but did not have totalis (sNEC).

\section{METHODS}

This was a multicenter retrospective cohort study with infants of all gestational ages receiving surgery for NEC identified in the CHND from January 2010 to December 2016. The CHND prospectively captures clinical data for infants admitted to 34 children's hospitals whose neonatal intensive care units have at least a level IV designation, more than 400 annual admissions, and more than 50\% of infants admitted are born outside the participating hospital [12]. Data collection methods and quality assurance measures have been published previously [3, 12]. Individual Institutional Review Boards (IRB) at participating centers approved participation in CHND, and the Emory University IRB exempted this study of retrospective analysis of de-identified data.

Infants admitted to a CHND center for the evaluation and treatment of NEC were identified. Those who had a diagnosis of NEC but never had surgery, had NEC surgery prior to admission to a CHND center, or whose NEC resolved prior to admission were excluded. To capture a similar clinical presentation and relative rapid surgical course for $t N E C$, we limited the cohort to infants who received surgery within five days of admission. As defined by the CHND, NEC requiring surgery was identified if an infant had clinical signs of NEC (bilious gastric aspirate or emesis, abdominal distention, or occult or gross blood in stool with no apparent rectal fissure) and radiographic findings (pneumatosis intestinalis, hepato-biliary gas, or pneumoperitoneum) and received one of the following surgeries: laparotomy, laparoscopy, ostomy creation for intestinal diversion, small bowel resection, large bowel resection, or primary intraperitoneal drain placement for treatment of NEC. Those with gastrointestinal anomalies including gastroschisis, omphalocele, bowel atresia, intestinal webs or stenosis, malrotation, and meconium ileus were excluded, as were infants with major or lethal congenital anomalies and those with cyanotic heart disease. Infants with focal intestinal perforation (FIP) identified at time of surgery, post-mortem, or with evidence of pneumoperitoneum without clinical signs of NEC were also excluded.

\footnotetext{
${ }^{1}$ Division of Neonatology, Department of Pediatrics, Emory University and Children's Healthcare of Atlanta, Atlanta, GA, USA. ${ }^{2}$ Children's Hospital Association, Lenexa, KS, USA.
} ${ }^{3}$ Division of Pediatric Surgery, Department of Surgery, Emory University School of Medicine, Atlanta, GA, USA. ${ }^{\circledR}$ email: atrose@emory.edu 
The study population was compared by the presence or absence of $t N E C$. $t N E C$, as defined in the CHND, was identified at the time of surgery or autopsy as such extensive large and small bowel involvement that the disease was considered lethal by the attending surgeon or pathologist respectively. The $s$ NEC group included those infants who had NEC surgery, as defined above, but did not have totalis. The study population was described using maternal, birth, and perinatal characteristics. Comorbidities at admission to CHND center were collected. Sepsis on admission was defined as a positive blood culture at the referring hospital or within $48 \mathrm{~h}$ of admission. Patent ductus arteriosus (PDA) was considered present prior to or at the time of referral if there was echocardiographic documentation from the referring center; pharmacologic treatment with indomethacin (prophylactic or treatment regimens), ibuprofen, or acetaminophen as well as surgical ligation were recorded. Perioperative characteristics, type of surgery, and post-surgical anatomy, when known, were collected. Short bowel syndrome/intestinal failure (SBS/IF) was identified when an infant had a major bowel resection $(<75 \mathrm{~cm}$ or $<50 \%$ of expected small bowel length remaining) and $>90$ days of parenteral nutrition. Mortality was assessed up to hospital discharge.

\section{Statistical analysis}

Data are presented as frequency and number for categorical variables and median and interquartile range (IQR) for continuous data. Comparisons of categorical variables used Chi-square test and Fisher's exact as appropriate. Continuous variables were compared with the non-parametric Wilcoxon Rank Sum Test as departure from normality was observed. Statistical testing was two-sided. Individual center $t$ NEC rate was calculated (percent of infants with NEC requiring surgery who had $t$ NEC). The Wilson Score was used to calculate $95 \%$ confidence intervals for population proportions. Survival analysis using Kaplan-Meier curves was conducted to evaluate time to death, and Logrank test was used to compare groups. $P$ value was considered significant at 0.05 . All statistical analysis was performed in SAS Enterprise Guide 7.1 (Cary, NC).

\section{RESULTS}

Of 119,105 CHND admissions during the study period, 4708 had a diagnosis of NEC. Of those with a NEC diagnosis, 1406 infants had a NEC surgery within five days of admission (Fig. 1). Those with congenital anomalies, focal intestinal perforation or surgery prior to admission were excluded. The remaining 1059 infants comprised the study group, of whom 898 (84.8\%) had sNEC and $161(15.2 \%)$ had $t$ NEC. Thirty-one of 34 CHND centers contributed patients to the study cohort. There were no statistically significant differences in demographic, perinatal and birth characteristics (Table 1). There was a preponderance of male infants, $60 \%$, which was not significantly different between the groups. The median gestational age and birth weight for the entire cohort were 27 weeks, (interquartile range (IQR) 25, 30 weeks) and 910 grams (IQR 700, 1382 grams), respectively; these were not different between $t$ NEC and $s N E C$ infants. The percentage of small for gestational age [13] infants was also not different between groups. Receipt of antenatal steroids was low in both groups ( $t$ NEC: $46.6 \%$, sNEC $47.2 \%$ ). $t$ NEC infants had a trend towards higher cesarean section rate, but the difference did not reach statistical significance.

NEC diagnosis occurred five days later in $t$ NEC infants (median 17 days [IQR 12, 17] vs 12 days [IQR 7, 25], $p<0.001$ ) but at a similar postmenstrual age. At the time of surgical referral, $t N E C$ infants were less likely to have severe IVH ( $t$ NEC $5.0 \%$ vs $s$ NEC $10.5 \%, p=0.029)$, to have received medical treatment for a PDA ( $t$ NEC $18.6 \%$ vs $s$ NEC $26.1 \%, p=0.048$ ) or to present with intestinal perforation ( $t$ NEC $5.6 \%$ vs $s N E C 13.1 \%, p=0.005$ ). There was no difference in the use of postnatal steroids. tNEC infants had greater need of intensive supportive care during transport and on arrival to referral centers including mechanical ventilation ( $t$ NEC $94.4 \%$ vs $s$ NEC $80.2 \%$ ), vasopressors ( $t$ NEC: $46.0 \%$ vs $s N E C: 24.7 \%$ ) and paralysis by neuromuscular blockade ( $t$ NEC $13 \%$ vs $s$ NEC $2.7 \%$ ) (all $p<0.001$ ). Thirteen percent of all infants had a positive blood culture within 3 days of NEC surgery, and this rate did not

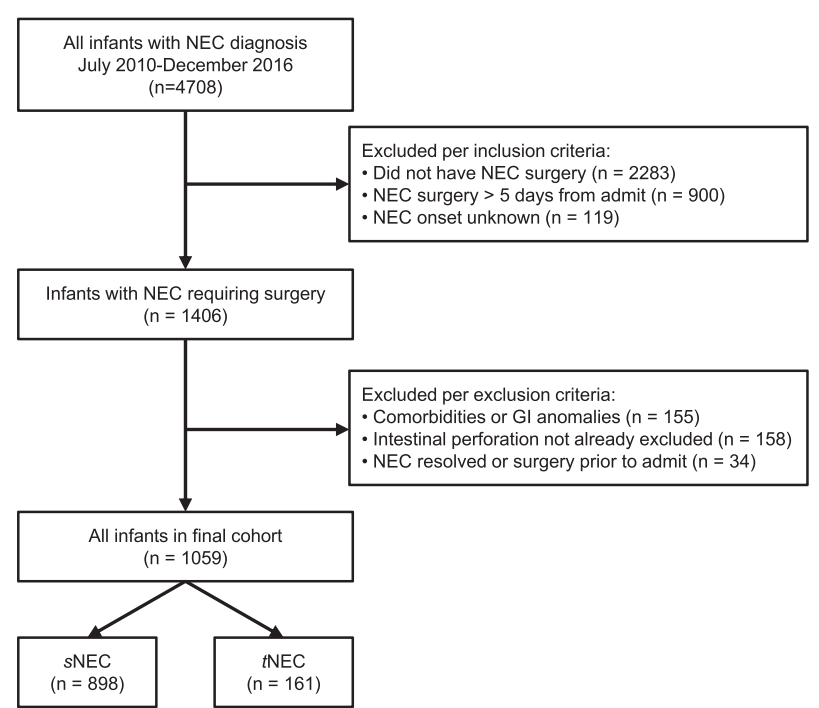

Fig. 1 Diagram of study population. NEC Necrotizing enterocolitis, Gl gastrointestinal, sNEC surgical necrotizing enterocolitis, tNEC necrotizing enterocolitis totalis.

differ between groups. Thirty percent of positive blood cultures were due to Escherichia coli and $23 \%$ to coagulase negative staphylococci.

The predominant surgery in both groups was a laparotomy. Only six (3.7\%) infants with $t$ NEC received a primary drain as the only surgical intervention (Table 2). Over half of sNEC infants were confirmed to have perforation at time of surgery, which was significantly more than their tNEC counterparts ( $t$ NEC $38.5 \%$ vs sNEC $66.7 \%, p<0.001)$. SBS/IF was diagnosed in $180(20.0 \%)$ of sNEC infants of whom $150(83 \%)$ survived hospital discharge. Of the 71 sNEC infants with documentation of remaining bowel length, the median was $50 \mathrm{~cm}$ (IQR 34-70). Fifteen tNEC infants received an ostomy, 26 had small bowel resection and 12 had large bowel resection. Of the surviving $t$ NEC patients, two had both large and small bowel resection, and two had just small bowel resection. However, none had documentation of length of bowel resected or length of bowel remaining. All were diagnosed with SBS/IF, and none were referred for bowel or liver transplant. No infant with $t$ NEC received only a primary drain and survived. Time from NEC diagnosis to surgery was significantly associated with mortality with a median time to surgery of 1 day (IQR $0-2)$ for survivors and 0 days (IQR $0-1$ ) for those who died, $p<0.001$. This finding remained true when only looking at infants with $s$ NEC $(p=0.016)$.

Overall mortality was $37 \%$ with a significant difference in time of death between groups (Fig. 2, logrank $p<0.001$ ). Over half of the $t$ NEC patients and a third of the sNEC patients died within $12 \mathrm{~h}$ of admission to a CHND center (Table 2). Intra-abdominal catastrophe was the primary cause of death in $94.2 \%$ of $t$ NEC infants, while one third of sNEC cases had other attributable primary cause of death (i.e., multi-organ system failure in 14\%, respiratory failure in $10 \%)$. Death or survival to discharge with SBS/ IF occurred in $546(51.6 \%)$ of all infants and in $388(43.2 \%)$ of $s$ NEC infants.

Twenty-seven out of 31 CHND centers contributed five or more patients to the entire study cohort; however, five centers had 10 or more $t$ NEC cases contributing $36.8 \%$ to the total cohort and $53.4 \%$ to the $t \mathrm{NEC}$ group. To understand center variation, we compared the proportion of $t$ NEC infants by center and cohort mortality by center (Fig. 3). The overall tNEC rate was 15\% (95\% Cl 13-17\%) which was unchanged after removing the four centers that contributed fewer than five patients to the total cohort. Across centers, the $t$ NEC rate varied from $0-43 \%$. One center had a statistically lower rate of $t$ NEC $(5 \%, 95 \%$ Cl 2-12\%) than for the 
Table 1. Demographic, birth and referral center admission characteristics for patients with sNEC and tNEC admitted to CHND centers between Jan 1, 2010 and Dec 31, 2016.

\begin{tabular}{|c|c|c|c|c|}
\hline Variable & All & sNEC & tNEC & $p$ \\
\hline Total (\%) & 1059 & $898(85)$ & $161(15)$ & - \\
\hline Gestational age (weeks) $^{\mathrm{a}}$ & $27(25,30)$ & $27(25,30)$ & $27(25,30)$ & 0.89 \\
\hline Birth weight (grams) ${ }^{a}$ & $910(700,1382)$ & $920(700,1400)$ & $870(715,1220)$ & 0.62 \\
\hline$S G A \leq 10 \%(\%)$ & $178(17)$ & $151(17)$ & $27(17)$ & $>0.99$ \\
\hline Female (\%) & $423(40)$ & $355(40)$ & $68(42)$ & 0.54 \\
\hline Maternal race (\%) & & & & 0.08 \\
\hline Black & $438(41)$ & $359(40)$ & $79(49)$ & \\
\hline Other & $122(11)$ & $103(12)$ & $19(12)$ & \\
\hline Hispanic (\%) & $136(13)$ & $119(13)$ & $17(11)$ & 0.55 \\
\hline \multicolumn{5}{|l|}{ Maternal conditions (\%) } \\
\hline Preterm labor & $445(42)$ & $382(43)$ & $63(39)$ & 0.44 \\
\hline \multicolumn{5}{|l|}{ Delivery room resuscitation (\%) } \\
\hline Endotracheal ventilation & $603(57)$ & $514(57)$ & $89(55)$ & 0.67 \\
\hline Epinephrine administration & $31(3)$ & $25(3)$ & $6(4)$ & 0.45 \\
\hline Cardiac compressions & $54(5)$ & $43(5)$ & $11(7)$ & 0.33 \\
\hline Cesarean delivery (\%) & $651(62)$ & $542(60)$ & $109(68)$ & 0.08 \\
\hline Antenatal steroids (\%) & $494(47)$ & $418(47)$ & $76(47)$ & 0.93 \\
\hline Postnatal steroids ${ }^{\mathrm{b}}(\%)$ & $436(41)$ & $370(41)$ & $66(41)$ & $>0.99$ \\
\hline Surfactant, any (\%) & $673(64)$ & $566(63)$ & $107(66)$ & 0.43 \\
\hline Age at NEC Diagnosis (days) ${ }^{a}$ & $13(7,25)$ & $12(7,24)$ & $17(12,27)$ & $<0.001$ \\
\hline PMA at NEC Diagnosis (weeks) ${ }^{a}$ & $30(27,33)$ & $30(27,33)$ & $30(29,32)$ & 0.55 \\
\hline
\end{tabular}

sNEC Surgical necrotizing enterocolitis without totalis, tNEC Necrotizing enterocolitis totalis, CHND Children's Hospitals Neonatal Database, IQR Interquartile range, SGA Small for gestational age, ROM Rupture of membranes, PMA Postmenstrual age.

a Median (25-75 percentile).

${ }^{b}$ Systemic steroids given prior to referral for any reason.

overall cohort and one had a higher rate $(43 \%, 95 \% \mathrm{Cl} 33-54 \%)$. The overall mortality rate was $37.2 \%$ ( $95 \% \mathrm{Cl} 34-40 \%)$, which was also unchanged after removing the four centers with fewer than five patients. The range for overall NEC mortality was $11-63 \%$ with two centers below and two centers above the overall cohort mortality rate (Fig. 3).

\section{DISCUSSION}

This study is the largest multicenter cohort of infants with $t$ NEC; in this group of infants who received surgery for NEC, we found $15 \%$ had $t$ NEC and $97 \%$ of those infants died. Infants with $t$ NEC were older at NEC diagnosis and less likely to present with bowel perforation than were infants without totalis. Given the significant burden of disease and catastrophic outcome, understanding differences in presentation and management for infants with $t$ NEC may help clinicians caring for these infants and their families.

Our findings add to the limited literature on $t$ NEC that was based primarily on retrospective studies by single institutions. Few consistent demographic variables, feeding data, or radiographic differences have been able to distinguish infants at-risk for $t$ NEC $[4,7,14]$. A retrospective analysis from a single-center designed to develop a predictive totalis-score was limited by small numbers (13 $t$ NEC cases) [8]. In our cohort, infants with $t$ NEC, although older at presentation, had findings consistent with severe illness including more metabolic acidosis and an increased need for ventilator and blood pressure support. Time to death after NEC diagnosis for $t$ NEC infants was significantly sooner than $s N E C$ infants. Interestingly, those with $t$ NEC had fewer common comorbidities of prematurity such as IVH and need for PDA treatment. Measures of illness severity, including preoperative inotrope use, mean blood pressure, and sepsis in addition to oneminute Apgar score were predictive of death or IF in a cohort of 147 surgical NEC patients [15]. The inclusion of one-minute Apgar score in this predictive model may be an attempt to account for unknown but important intrauterine factors. However, our larger cohort did not find differences in maternal conditions including chorioamnionitis, prolonged rupture of membranes, maternal hypertensive diseases, or placental abruption. We also found no difference in delivery room interventions including endotracheal intubation, epinephrine administration, or cardiac compressions. There was a trend towards a difference in cesarean section rates with a non-statistically significant higher rate in $t$ NEC infants.

A unique finding in our study was the lack of perforation in the $t$ NEC compared to $s$ NEC infants, both at time of admission as recorded in admitting diagnoses as well as at time of surgery. Bowel perforation remains the clearest indication for surgery in infants with NEC, while declining physiologic state, portal venous gas, and abdominal wall erythema are relative indications [16]. The decision to proceed with surgery in $t$ NEC may have been related 
Table 2. Perioperative, surgical characteristics and mortality of patients with sNEC and tNEC.

\begin{tabular}{|c|c|c|c|c|}
\hline Variable & All & sNEC & $t$ NEC & $p$ \\
\hline Total (\%) & 1059 & $898(85)$ & $161(15)$ & - \\
\hline Age at NEC surgery (days) ${ }^{a}$ & $14(7,26)$ & $13(7,26)$ & $18(12,27)$ & 0.001 \\
\hline PMA at NEC surgery (weeks) ${ }^{a}$ & $30(28,33)$ & $30(27,33)$ & $30(29,32)$ & 0.76 \\
\hline Days from diagnosis to surgery ${ }^{a}$ & $0(0,1)$ & $0(0,1)$ & $0(0,2)$ & 0.001 \\
\hline Perioperative bacteremia ${ }^{\mathrm{b}}(\%)$ & $140(13)$ & $119(13)$ & $21(13)$ & $>0.99$ \\
\hline Preoperative $\mathrm{pH}^{\mathrm{a}, \mathrm{c}}$ & $7.3(7.2,7.3)$ & $7.3(7.2,7.4)$ & $7.1(7.0,7.3)$ & $<0.001$ \\
\hline Preoperative glucose $\mathrm{e}^{\mathrm{a}, \mathrm{c}}$ & $102(79,146)$ & $101(79,140)$ & $109(76,186)$ & 0.12 \\
\hline NEC surgery type & & & & $<0.001$ \\
\hline Laparotomy (\%) & $759(72)$ & $627(70)$ & $132(82)$ & \\
\hline Primary drain (\%) & $159(15)$ & $146(16)$ & $6(4)$ & \\
\hline Laparotomy and drain (\%) & 139 (13) & $116(13)$ & $23(14)$ & \\
\hline Transfer for transplant (\%) & $2(0.2)$ & $2(0.2)$ & $0(-)$ & $>0.99$ \\
\hline Mortality (\%) & $392(37)$ & $238(27)$ & $156(97)$ & $<0.001$ \\
\hline Death $\leq 12 \mathrm{~h}$ from admission (\%) & $163(41)$ & $81(34)$ & $82(53)$ & $<0.001$ \\
\hline \multicolumn{5}{|c|}{$\begin{array}{l}\text { sNEC Surgical necrotizing enterocolitis without totalis, } t N E C \text { Necrotizing enterocolitis totalis, IQR interquartile range, } P M A \text { Postmenstrual age, SBS Short bowel } \\
\text { syndrome/intestinal failure. } \\
\text { a Median (25th-75th percentiles) } \\
\text { bPositive culture within three days of surgical procedure } \\
{ }^{c} \text { Number missing data for preoperative labs included: } s N E C: 109 \mathrm{pH}, 109 \mathrm{pCO}_{2}, 123 \text { glucose. tNEC: } 12 \mathrm{pH}, 13 \mathrm{pCO}_{2}, 23 \text { glucose }\end{array}$} \\
\hline
\end{tabular}

to clinical indications such as declining physiologic state as evidenced by more severe acidosis found in this group. A recurrent problem in NEC studies, especially in those focusing on surgical NEC, is the difficulty in excluding infants with FIP. Placement of a primary peritoneal drain is a management approach to both entities, and, without additional surgery, it precludes direct inspection of the bowel. To address this bias, we excluded infants who were diagnosed with FIP at surgery or autopsy, as well as those presumed to have FIP as determined by the care team based on the infant's clinical course. Our methodology was similar to prior studies of surgical NEC patients $[3,17]$. However, contamination with FIP is still possible, as $25 \%$ of infants with $s N E C$ were diagnosed at a week or younger of age, a timeframe more consistent with FIP [18]. By limiting the cohort to infants having a surgical procedure within 5 days of admission, we hoped to allow for a clinically relevant comparison of $t$ NEC and sNEC hospital and surgical courses while excluding infants who may have had equivocal decisions for surgery. We thought it highly unlikely a patient with tNEC would have a surgical intervention beyond five days from admission.

$t N E C$ is poorly defined as a clinical entity in the literature. A recent systematic review on pediatric $t$ NEC found that over $50 \%$ of published reports had no clear definition of $t \mathrm{NEC}$, and those that did define the disease had variable definitions [2]. Consortiums like the CHND can spearhead collaborative efforts to come to consensus on topics like $t$ NEC definitions. Despite a codified definition of $t N E C$ in CHND, it does allow for a fair degree of subjectivity, which may be reflected by the variability of tNEC cases across CHND centers. Center rates of $t$ NEC in our cohort varied widely with rates ranging from zero to $43 \%$. The subjective nature of the definition of $t$ NEC is vulnerable to bias both between and within centers as it is defined by the individual surgeon. However, subjectivity likely does not explain all center variation. Institutional practice, variations in timing and distance for transport, as well as regional differences in NEC prevalence and mortality may be involved [19].

Aggressive surgical therapy in tNEC patients was associated with decreased mortality in a literature review of 414 published cases [2]. As all pediatric cases of $t$ NEC were included in the review, it is not clear that the improvement in mortality found with aggressive surgical management would translate to a neonatal population, especially one involving extremely premature infants who may have other significant comorbidities. An analogy to the infant with $t$ NEC may be the decision regarding resuscitation of infant born at 22 weeks of gestational age. Hospital rates of initiating active treatment in infants born at 22 weeks account for variation between hospitals in survival and survival without neurodevelopmental impairment in these infants [20]. Perhaps there is a subgroup of $t$ NEC patients who, with intervention, have potential for survival given the risk balance for long-term survival with SBS/IF after NEC [21, 22]. Clinicians have mixed opinions on management approaches to infants with minimal residual bowel length after NEC $[9,10]$. While up to $90 \%$ of neonatologists and surgeons think offering life-sustaining interventions to infants with $t$ NEC is ethically permissible, $71 \%$ would not recommend it [10]. While there have been significant improvements in the care of patients with SBS/IF, few $t N E C$ patients have survived beyond discharge thus limiting information on their long-term outcomes [11]. Little is known about parents' perspective about treatment options and important outcome measures as they relate to NEC much less $t$ NEC $[23,24]$. As clinicians question the management of these patients and consider possible interventions, parents' perspective and input should be sought. While our findings that patients with tNEC are less likely to have pneumoperitoneum and have a lower $\mathrm{pH}$ are not intended to be predictive of $t$ NEC as an outcome, they could help physicians in preoperative counseling of families that would then lay the groundwork for future discussion of goals of care. 


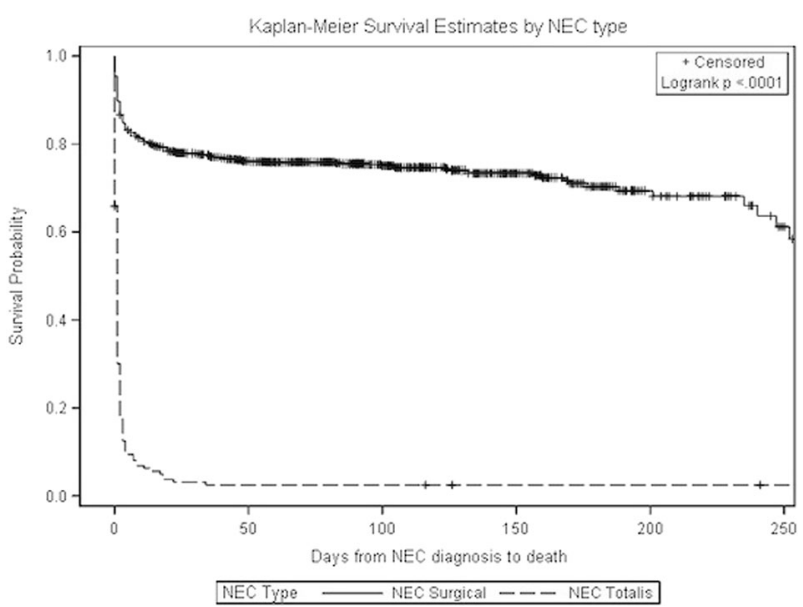

Fig. 2 Kaplan-Meier Survival curves by necrotizing enterocolitis typic. Time to death is significantly different between tNEC and sNEC infants. (Logrank $p<0.001$ ).

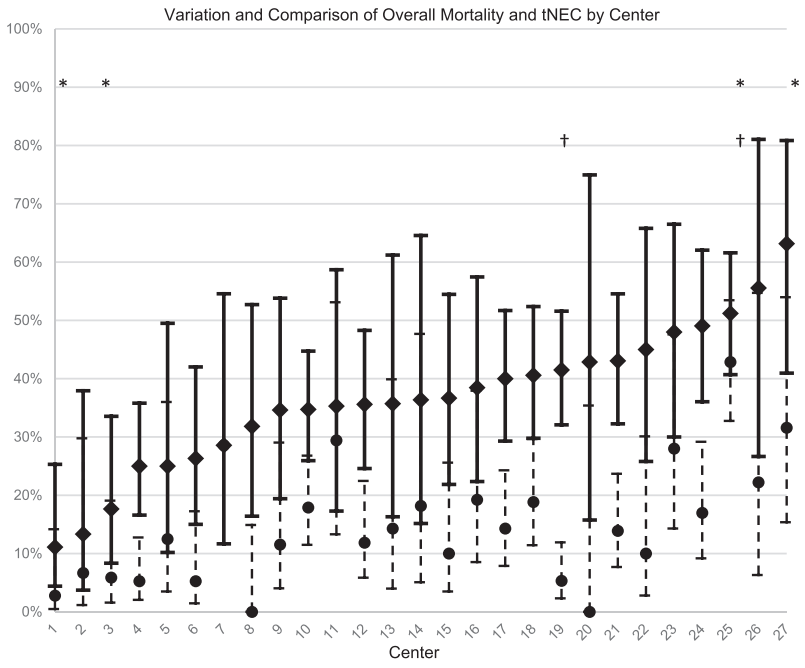

Fig. 3 Percent overall mortality by center and percent totalis by center for centers contributing at least five patients to the study cohort. The $x$ axis represents individual CHND centers ordered by percent mortality. Black diamonds represent the percentage of all infants (tNEC + sNEC) who died by center with $95 \%$ confidence intervals $(95 \% \mathrm{Cl})$. Black dots represent the percentage of infants with tNEC by center with $95 \% \mathrm{Cl}$. ${ }^{*}$ Centers whose mortality rate is different than full cohort mortality rate $(37.2 \%, 95 \% \mathrm{Cl} 34-40 \%)$. tCenters whose totalis rate is different than full cohort totalis rate (15\%, 95\% Cl 13-17\%).

Despite leveraging the strengths of a national consortium, there are some important limitations to this study, including those associated with referral bias. This cohort includes those infants stable enough for transport to a CHND center, and thus may not include infants with severe NEC or $t$ NEC who cannot be moved. In addition, the database is limited on pre-admission feeding data such as human milk feeding, feeding volumes, feeding advancement rates, and probiotics; this information may shed light on risk factors or protective factors for $t N E C$. Of the 180 infants diagnosed with SBS/IF, less than half had documentation of bowel length remaining. The CHND implements rigorous data quality checks and thus our limited data may be related to variations in surgical documentation at centers. This finding leaves room for multicenter quality improvement efforts to improve surgical documentation in infants with surgical NEC and codify a clearer definition of $t$ NEC. As discussed earlier, despite our best efforts, the clinical overlap between FIP and sNEC may have resulted in some FIP cases in the $s N E C$ group. It is less likely the $t$ NEC group had contamination given the low rate of peritoneal drain as the sole operative management approach. And finally, the database is limited in granular data such as specific laboratory values perhaps contributing to the lack of indefinable clinical markers to help identify at-risk patients, leading to an opportunity for further potential for collaboration across centers.

$t$ NEC is a rare disease in neonates; however, in those infants referred for surgical evaluation due to NEC, it can be present in $15 \%$ of cases. In our population, infants with $t$ NEC were older at presentation and were less likely to have an intestinal perforation. The rates of $t \mathrm{NEC}$ varied by center, and this variation may be related to differences in practice and the subjective nature of $t$ NEC definition. Collaborative efforts are needed to uniformly document surgical procedures and agreed upon definitions in order to impact practices and outcomes. Currently tNEC consistently results in almost definitive mortality; but, as clinicians deliberate alternative interventions other than palliation, with improved gastrointestinal rehabilitation and engagement of parents, the potential for postdischarge survival may be possible in certain infants.

\section{REFERENCES}

1. Jacob J, Kamitsuka M, Clark RH, Kelleher AS, Spitzer AR. Etiologies of NICU deaths. Pediatrics. 2015;135:e59-65.

2. Dukleska K, Devin CL, Martin AE, Miller JM, Sullivan KM, Levy C, et al. Necrotizing enterocolitis totalis: high mortality in the absence of an aggressive surgical approach. Surgery. 2019;165:1176-81.

3. Murthy K, Yanowitz TD, DiGeronimo R, Dykes FD, Zaniletti I, Sharma J, et al. Shortterm outcomes for preterm infants with surgical necrotizing enterocolitis. J Perinatol. 2014;34:736-40.

4. Voss M, Moore SW, van der Merwe I, Pieper C. Fulminanting necrotising enterocolitis: outcome and prognostic factors. Pediatr Surg Int. 1998;13:576-80.

5. Clark RH, Gordon P, Walker WM, Laughon M, Smith PB, Spitzer AR. Characteristics of patients who die of necrotizing enterocolitis. J Perinatol. 2012;32:199-204.

6. Rose AT, Patel RM. A critical analysis of risk factors for necrotizing enterocolitis. Semin Fetal Neonatal Med. 2018;23:374-9.

7. Thompson A, Bizzarro M, Yu S, Diefenbach K, Simpson BJ, Moss RL. Risk factors for necrotizing enterocolitis totalis: a case-control study. J Perinatol. 2011;31:730-8.

8. Sho S, Neal MD, Sperry J, Hackam DJ. A novel scoring system to predict the development of necrotizing enterocolitis totalis in premature infants. J Pediatr Surg. 2014;49:1053-6.

9. Pet GC, McAdams RM, Melzer L, Oron AP, Horslen SP, Goldin A, et al. Attitudes surrounding the management of neonates with severe necrotizing enterocolitis. J Pediatr. 2018;199:186-93.e183.

10. Pyle AK, Shabanova V, Cleary MA, Ozgediz D, Cummings CL, Kamin DS, et al. Variable management strategies for NEC totalis: a national survey. J Perinatol. 2019;39:1521-7.

11. Han SM, Knell J, Henry O, Riley H, Hong CR, Staffa SJ, et al. Long-term outcomes of severe surgical necrotizing enterocolitis. J Pediatr Surg. 2020;55:848-51.

12. Murthy K, Dykes FD, Padula MA, Pallotto EK, Reber KM, Durand DJ, et al. The Children's Hospitals Neonatal Database: an overview of patient complexity, outcomes and variation in care. J Perinatol. 2014;34:582-6.

13. Olsen IE, Groveman SA, Lawson ML, Clark RH, Zemel BS. New intrauterine growth curves based on United States data. Pediatrics. 2010;125:e214-24.

14. Moss RL, Kalish LA, Duggan C, Johnston P, Brandt ML, Dunn JCY, et al. Clinical parameters do not adequately predict outcome in necrotizing enterocolitis: a multi-institutional study. J Perinatol. 2008;28:665-74.

15. Bhatt D, Travers C, Patel RM, Shinnick J, Arps K, Keene S, et al. Predicting mortality or intestinal failure in infants with surgical necrotizing enterocolitis. J Pediatr. 2017;191:22-27.e23.

16. Henry MC, Lawrence, Moss R. Surgical therapy for necrotizing enterocolitis: bringing evidence to the bedside. Semin Pediatr Surg. 2005;14:181-90.

17. Yanowitz TD, Sullivan KM, Piazza AJ, Brozanski B, Zaniletti I, Sharma J, et al. Does the initial surgery for necrotizing enterocolitis matter? Comparative outcomes for laparotomy vs. peritoneal drain as initial surgery for necrotizing enterocolitis in infants $<1000 \mathrm{~g}$ birth weight. J Pediatr Surg. 2019;54:712-7.

18. Gordon PV, Clark R, Swanson JR, Spitzer A. Can a national dataset generate a nomogram for necrotizing enterocolitis onset? J Perinatol. 2014;34:732-5.

19. Wolf MF, Rose AT, Goel R, Canvasser J, Stoll BJ, Patel RM. Infant Mortality in the United States due to Necrotizing Enterocolits, 1999-2017: Trends and Racial \& 
Geographic Disparities (abstract 3378532). Pediatric Academic Societies 2020; Philadelphia, PA.

20. Rysavy MA, Li L, Bell EF, Das A, Hintz SR, Stoll BJ, et al. Between-hospital variation in treatment and outcomes in extremely preterm infants. N. Engl J Med. 2015;372:1801-11.

21. Sparks EA, Khan FA, Fisher JG, Fullerton BS, Hall A, Raphael BP, et al. Necrotizing enterocolitis is associated with earlier achievement of enteral autonomy in children with short bowel syndrome. J Pediatr Surg. 2016;51:92-95.

22. Khan FA, Squires RH, Litman HJ, Balint J, Carter BA, Fisher JG, et al. Predictors of enteral autonomy in children with intestinal failure: a multicenter cohort study. J Pediatr. 2015;167:29-34.e21.

23. Gadepalli SK, Canvasser J, Eskenazi Y, Quinn M, Kim JH, Gephart SM. Roles and experiences of parents in necrotizing enterocolitis: an international survey of parental perspectives of communication in the NICU. Adv Neonatal Care. 2017;17:489-98.

24. Webbe JWH, Duffy JMN, Afonso E, Al-Muzaffar I, Brunton G, Greenough A, et al. Core outcomes in neonatology: development of a core outcome set for neonatal research. Arch Dis Child Fetal Neonatal Ed. 2020;105:425-31.

\section{ACKNOWLEDGEMENTS}

We are most grateful to Ira Adams-Chapman, MD. She contributed extensively with her fund of knowledge and professional mentorship throughout this project including concept, design, and editing until her premature death on October 27, 2020. We thank Karna Murthy, MD (Ann \& Robert H. Lurie Children's Hospital of Chicago) for his ongoing critical review of this project and Ravi M. Patel, MD, MSc (Emory University School of Medicine) for his critical review of this manuscript. We thank Shira M. Asherman (Emory University School of Medicine) for her editing. We are indebted to the clinicians and institutions that serve the infants and families of the Children's Hospital's Neonatal Consortium.

\section{AUTHOR CONTRIBUTIONS}

ATR and AJP conceptualized and designed the study, interpreted the results, drafted the initial manuscript, reviewed and revised the manuscript, and participated in funding acquisition. IZ participated in study design, data analysis, and reviewed and revised the manuscript. MTS conceptualized and designed the study, reviewed and revised the manuscript. All authors approved the final manuscript as submitted and agree to be accountable for all aspects of the work.

\section{FUNDING}

Supported by Department of Pediatrics at Emory University Fellowship Grant $(<20205>$ [to Allison T. Rose, MD]).

\section{COMPETING INTERESTS}

All listed authors declare no real or perceived conflicts of interest with the exception of Isabella Zaniletti, who is an employee of the Children's Hospital Association, Inc (Overland Park, KS) which designed, launched and maintained the database used in this study from 2012-2016.

\section{ADDITIONAL INFORMATION}

Correspondence and requests for materials should be addressed to A.T.R.

Reprints and permission information is available at http://www.nature.com/ reprints

Publisher's note Springer Nature remains neutral with regard to jurisdictional claims in published maps and institutional affiliations. 